\title{
Deformation and orientation effects in heavy-particle radioactivity of $Z=115$
}

\author{
Gudveen Sawhney ${ }^{1, a}$, Kirandeep Sandhu ${ }^{2}$, Manoj K. Sharma ${ }^{2}$, and Raj K. Gupta ${ }^{1}$ \\ ${ }^{1}$ Department of Physics, Panjab University, Chandigarh-160014, India \\ ${ }^{2}$ School of Physics and Materials Science, Thapar University, Patiala-147004, India
}

\begin{abstract}
The possibility of heavy particle radioactivity (heavier clusters) in ground state decays of ${ }^{287-289} 115$ parent nuclei, resulting in a doubly magic daughter around ${ }^{208} \mathrm{~Pb}$ is analyzed using Preformed Cluster Model (PCM) with choices of spherical and quadrupole deformation $\left(\beta_{2}\right)$ having "optimum" orientations of decay products. The behavior of fragmentation potential and preformation probability is investigated in order to extract better picture of the dynamics involved. Interestingly, the potential energy surfaces obtained via the fragmentation process get modified significantly with the inclusion of deformation and orientation effects, which in turn influence the preformation factor.
\end{abstract}

\section{Introduction}

The radioactive decay of nuclei emitting particles heavier than alpha-particle, predicted in 1980 [1], was confirmed in 1984 [2] via the ${ }^{14} \mathrm{C}$ decay from ${ }^{223} \mathrm{Ra}$ nucleus. With this discovery, a big hunt for more and more cluster emitters was stimulated and as a result today we have a family of cluster radioactive decays leading to ${ }^{12,14} \mathrm{C},{ }^{15} \mathrm{~N},{ }^{18,20} \mathrm{O}$, ${ }^{23} \mathrm{~F},{ }^{22,24-26} \mathrm{Ne},{ }^{28,30} \mathrm{Mg}$, and ${ }^{32,34} \mathrm{Si}$ emissions. To date, ${ }^{34} \mathrm{Si}$ is the heaviest cluster observed with the longest decay half-life $\left(\log _{10} T_{1 / 2}(s)=29.04\right)$ from ${ }^{238} \mathrm{U}$ parent, and the smallest branching ratio of cluster w.r.t. $\alpha$-decay, $B$ $=\lambda_{\text {cluster }} / \lambda_{\alpha} \sim 10^{-17}$ for ${ }^{28,30} \mathrm{Mg}$ decay of ${ }^{238} \mathrm{Pu}$ [3]. All the cluster emitters studied so far, belong to trans-lead region, giving closed shell ${ }^{208} \mathrm{~Pb}$ or its neighboring nuclei as residual or daughter nucleus. The cluster emission and related aspects have been studied extensively using various models during last three decades.

The exploration of cluster radioactivity in the superheavy (SHE) region did not receive much attention because of the instability of nuclei in this region. Besides beta decay, only $\alpha$-decay and spontaneous fission of SHE nuclei have been experimentally observed up to now. Knowing that the role of shell effects is the central feature in the cluster decay process studied so far, the domain of cluster radioactivity has been further widened by Poenaru et al. $[4,5]$. They explored the heavy-particle radioactivity of superheavy nuclei on the basis of Analytical Super Asymmetric Fission Model (ASAFM) in which unstable parent nuclei having $Z>110$ decays into a cluster with $Z_{\text {cluster }}>28$ and a doubly magic daughter around ${ }^{208} \mathrm{~Pb}$. As a follow up of this work, we have studied in this paper the ground state decays of ${ }^{289} 115,{ }^{288} 115$, and ${ }^{287} 115$ SHE systems using the Preformed Cluster Model (PCM) $[6,7]$. These systems have been observed [8] in 2n, 3n and

\footnotetext{
${ }^{\mathrm{a}}$ e-mail: gudveen.sahni@gmail.com
}

$4 \mathrm{n}$-evaporation channels produced in a fusion reaction of ${ }^{48} \mathrm{Ca}$ beam with the ${ }^{243} \mathrm{Am}$ target.

The PCM finds its basis in the well known Quantum Mechanical Fragmentation Theory (QMFT) where the cluster is assumed to be preformed in the mother nucleus and the preformation probability (also known as spectroscopic factor) for all possible clusters are calculated by solving the Schrödinger equation for the dynamic flow of mass and charge. In view of the excellent agreement $[9,10]$ of PCM with the available $[11,12]$ experimental data on cluster decays of heavy parent nuclei with $Z=87$ to 96 , here in this work, half lives of isotopes of SHE element $Z=115$ have been predicted and compared with the existing $[4,5]$ theoretical results to test the extent of validity of this formalism. Since the fragmentation process depends on the collective clusterization approach, in PCM, not only the shapes of parent, daughter and cluster are important but also of all other possible fragments anticipated in the decay. It is expected that, together with shell effects, nuclear deformations and orientations also play an important role in the cluster decay process. In order to look for such effects, we intend to investigate the role of spherical as well as quadrupole $\left(\beta_{2}\right)$ deformations on the behavior of possible fragmentations of the decaying parent nucleus. It may be noted that deformation effects up to quadrupole $\beta_{2}$ are included with in the "optimum" orientation [13] approach. However, if one is interested in investigating the role of higher order deformations then "compact" orientations [7] should be preferred instead of "optimum" [13] orientations.

The paper is organized as follows: Sections 2 and 3 give, respectively, the details of the Preformed Cluster Model and our calculations for ground state decays of the chosen parent nuclei. Finally, the results are summarized in Section 4. 


\section{The Preformed Cluster Model (PCM)}

In PCM, we use the collective coordinates of mass and charge asymmetries, and relative separation $R$ which allow to define the decay constant $\lambda$, and hence the decay halflife time $T_{1 / 2}$, as

$$
\lambda=v_{0} P_{0} P, \quad T_{1 / 2}=\frac{\ln 2}{\lambda}
$$

$P_{0}$ is the cluster preformation probability and $P$ is the barrier penetrability which refer, respectively, to the $\eta$ and $R$-motions, both depending on multipole deformations $\beta_{\lambda i}$ and orientations $\theta_{i}(i=1,2)$ of the daughter and cluster nuclei. Here $v_{0}$ is the assault frequency with which the cluster hits the barrier, given by

$$
v_{0}=\frac{v}{R_{0}}=\frac{\left(2 E_{2} / \mu\right)^{1 / 2}}{R_{0}}
$$

$P_{0}$ is the solution of the stationary Schrödinger equation in $\eta$ given by

$$
\left[-\frac{\hbar^{2}}{2 \sqrt{B_{\eta \eta}}} \frac{\partial}{\partial \eta} \frac{1}{\sqrt{B_{\eta \eta}}} \frac{\partial}{\partial \eta}+V_{R}(\eta)\right] \psi^{\omega}(\eta)=E^{\omega} \psi^{\omega}(\eta)
$$

which on proper normalization gives

$$
P_{0}=\left|\psi\left[\eta\left(A_{i}\right)\right]\right|^{2} \sqrt{B_{\eta \eta}} \frac{2}{A_{C N}},
$$

with $i=1,2$ and $\omega=0,1,2,3 \ldots$ referring to ground-state $(\omega=0)$ and excited-states solutions.

The fragmentation potential $V_{R}(\eta)$ in Eq. (3) is calculated simply as the sum of Coulomb interaction, the nuclear proximity, angular-momentum dependent potentials and the ground state binding energies of two nuclei:

$$
\begin{aligned}
V_{R}(\eta) & =-\sum_{i=1}^{2}\left[B_{i}\left(A_{i}, Z_{i}\right)\right]+V_{C}\left(R, Z_{i}, \beta_{\lambda i}, \theta_{i}\right) \\
& +V_{P}\left(R, A_{i}, \beta_{\lambda i}, \theta_{i}\right)+V_{\ell}\left(R, A_{i}, \beta_{\lambda i}, \theta_{i}\right) .
\end{aligned}
$$

with $B^{\prime} s$ taken from experimental data of Audi-Wapstra [14] and wherever not available, the theoretical values of Möller et al. [15] are used. The deformation parameters $\beta_{\lambda i}$ of nuclei are also taken from [15]. Thus, shell effects are contained in our calculations that come from the experimental and/or calculated binding energies. For ground state decays, $\ell=0$ is a good approximation [11].

The penetrability $P$ in Eq. (1) is the WKB integral between the two turning points $R_{a}$ and $R_{b}$ and is given by $P=P_{i} P_{b}$, where $P_{i}$ and $P_{b}$, in WKB approximation, are

$$
P_{i}=\exp \left[-\frac{2}{\hbar} \int_{R_{a}}^{R_{i}}\left\{2 \mu\left[V(R)-V\left(R_{i}\right)\right]\right\}^{1 / 2} d R\right]
$$

and

$$
P_{b}=\exp \left[-\frac{2}{\hbar} \int_{R_{i}}^{R_{b}}\{2 \mu[V(R)-Q]\}^{1 / 2} d R\right]
$$

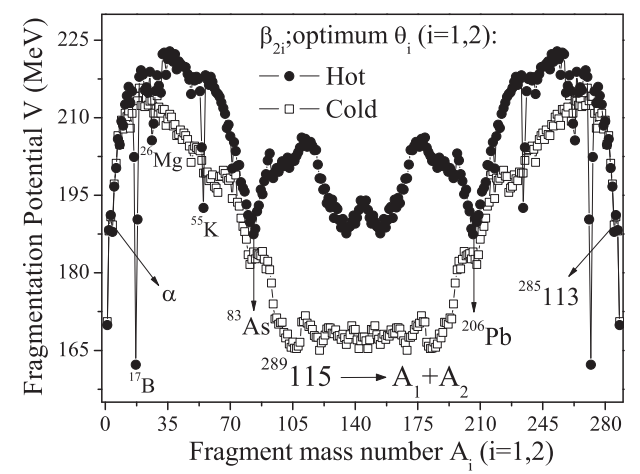

Figure 1: Fragmentation potential for the parent nucleus ${ }^{289} 115$ with quadrupole deformation $\beta_{2}$ and "optimum" orientations forming hot (compact) and cold (non-compact) configurations.

For the first turning point $R_{a}$, we use the postulate

$$
\begin{aligned}
R_{a}(\eta) & =R_{1}\left(\alpha_{1}\right)+R_{2}\left(\alpha_{2}\right)+\Delta R \\
& =R_{t}(\alpha, \eta)+\Delta R
\end{aligned}
$$

where the $\eta$-dependence of $R_{a}$ is contained in $R_{t}$, and $\Delta R$ is a parameter, assimilating the neck formation effects of two centre shell model shape. In the above equations (5) and $(8), \theta_{i}$ is the orientation angle between the nuclear symmetry axis and the collision $Z$ axis, measured in the anticlockwise direction, and angle $\alpha_{i}$ is the angle between the symmetry axis and the radius vector $R_{i}$ of the colliding nucleus, measured in the clockwise direction from the symmetry axis (see, e.g., Fig. 1 of Ref. [13]).

The nuclear proximity potential in Eq. (5) for deformed, oriented nuclei [16], used in the present work, is referred as Prox 2000 and given by

$$
V_{p}\left(s_{0}\right)=4 \pi \bar{R} \gamma b \Phi\left(s_{0}\right),
$$

where $b=0.99$ is the nuclear surface thickness, $\gamma$ is the surface energy constant and $\bar{R}$ is the mean curvature radius (for details, see Ref. [16]). $\Phi$ in Eq. (9) is the universal function, independent of the shapes of nuclei or the geometry of the nuclear system, but depends on the minimum separation distance $s_{0}$. The universal function is taken from Myers and Swiatecki [17], as

$$
\Phi\left(s_{0}\right)=\left\{\begin{array}{l}
-0.1353+\sum_{n=0}^{5}\left[c_{n} /(n+1)\right]\left(2.5-s_{0}\right)^{n+1} \\
-0.09551 \exp \left[\left(2.75-s_{0}\right) / 0.7176\right]
\end{array}\right.
$$

for $0<s_{0} \leq 2.5$ and $s_{0} \geq 2.5$, respectively, where $s_{0}=$ $R-R_{1}-R_{2}$. The values of different constants $c_{n}$ are $c_{0}$ $=-0.1886, c_{1}=-0.2628, c_{2}=-0.15216, c_{3}=-0.04562$, $c_{4}=0.069136$, and $c_{5}=-0.011454$. For further details of surface energy coefficient and nuclear charge radius, etc., see Ref. [17].

\section{Calculations and results}

The analysis of cluster radioactivity using PCM, previously associated with atomic number $\left(2<Z_{\text {cluster }}<20\right)$, is now extended up to the heavier cluster $\left(Z_{\text {cluster }}^{\max } \sim Z_{\text {parent }}{ }^{-}\right.$ 82 ) in order to get information about the most probable 


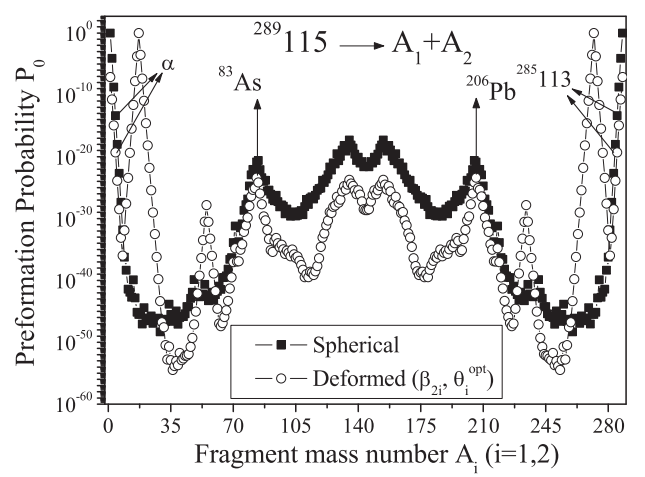

Figure 2: PCM calculated preformation probability $P_{0}$ for the decay of ${ }^{289} 115$ with the spherical and $\beta_{2}$ deformed choices of fragmentation.

heavy cluster and a doubly magic daughter around ${ }^{208} \mathrm{~Pb}$ for the isotopes of superheavy element $Z=115$. Figure 1 shows the fragmentation potential of the parent nucleus ${ }^{289} 115$ for the case of quadrupole deformation $\beta_{2}$ and "optimum" orientations [13] taken into account for all the possible fragments. The "optimum" orientations $\theta^{\text {opt }}$ are uniquely fixed [13] on the basis of quadrupole deformations $\beta_{2 i}$ of nuclei alone which manifest in the form of "hot (compact)" and "cold (non-compact)" configuration. The 'hot compact' configuration corresponds to smallest interaction radius and highest barrier, whereas the 'cold non-compact' configuration corresponds to largest interaction radius and lowest interaction barrier. The calculations are done at inter-nuclear separation distance (equivalently, the neck length parameter $\Delta R$ ) shown in Table 1. The fragmentation potential plotted in Figure 1 depicts the comparative behavior of 'hot (compact)' and 'cold (noncompact)' orientations. A solid vertical line is drawn in order to point out the ${ }^{83}$ As cluster emitted from the ${ }^{289} 115$ parent nucleus. Also, some extra valleys at ${ }^{17} \mathrm{~B},{ }^{26} \mathrm{Mg}$ and ${ }^{55} \mathrm{~K}$ fragments are observed, but they get ruled out in calculations due to their negligible penetrability $P$ values. Thus, using PCM, the decay characteristics of ${ }^{289} 115$ nucleus clearly show that ${ }^{83} \mathrm{As}$ is most probable heavy particle with corresponding ${ }^{206} \mathrm{~Pb}$ daughter for the choice of "optimum" orientations of 'compact hot' configuration. In other words, region for heavy particle radioactivity is more favorable (minimum potential energy) and hence show a clear preference for 'hot compact' in comparison to 'cold non-compact' configuration. In reference to the above observation, further investigations are done by using 'compact hot' configurations only.

To investigate the possible role of deformations further, Figure 2 shows the variation of the preformation probability $P_{0}$ for the decay of ${ }^{289} 115$ as a function of fragment mass $A_{i}$, for both the cases, i.e., fragments taken as spheres (solid squares) and with quadrupole deformation $\beta_{2}$ (open circles) within the optimum "hot" orientation approach. The preformation probability $P_{0}$ of the fragments (before tunneling through the barrier) accounts for the structure effects in the decay process of a nuclear system. We find that the inclusion of deformation and orientation effects of the decaying fragments changes the relative

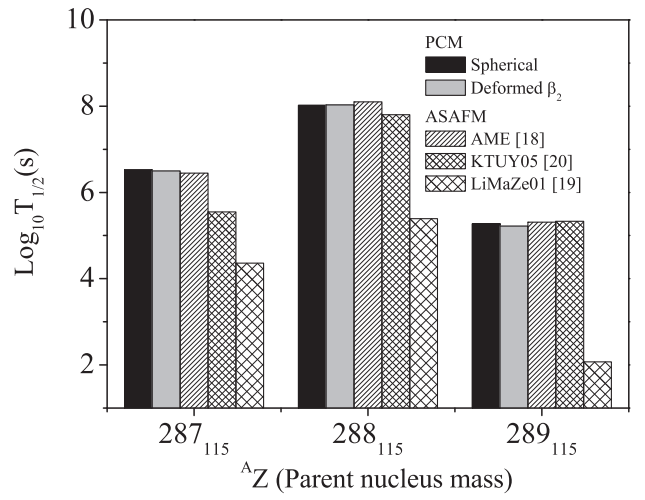

Figure 3: The decay half-lives for the most probable clusters emitted from various isotopes of $Z=115$ nuclei, with $(\mathrm{a})^{204} \mathrm{~Pb}(\mathrm{~b})^{205} \mathrm{~Pb}$ and (c) ${ }^{206} \mathrm{~Pb}$ daughter, calculated on the basis of PCM and compared with those calculated on ASAFM, plotted as a function of parent nucleus mass.

preformation probability $P_{0}$, quite significantly which in turn affects the decay constant and half life time accordingly. Despite the change in PES, the most probable cluster in Figure 2 remains the same, i.e., ${ }^{83}$ As cluster seems equally favoured for both the choices. It is important to note here that $A \sim 80$ is most often taken as a limiting light fission fragment, however we are considering it as heavy cluster decay. One can clearly see from Figure 2 that $\alpha$ particle is preferred over mass 83 fragment. The other two peaks at $A=17$ and 55 are ruled out by $P$ value being small. Similarly, barrier position as well as its height (not shown here) are also modified with deformation and orientation effects of outgoing fragments included, thereby affecting $P$.

The relevant details of preformation probabilities $P_{0}$, penetrability $P$, and assault frequencies $v_{0}$ for the most probable cluster decays of the considered parents with spherical and deformed choices of fragmentation using PCM are given in Table 1. We observe that preformation probability $P_{0}$ decreases and penetrability $P$ increases while going from spherical to deformed fragmentation. On the other hand, assault frequency $v_{0}$ remains almost constant, independent of choice of deformation effects. Apparently, as both $P_{0}$ and $P$ are affected by the inclusion of deformation and orientation effects, the calculated $T_{1 / 2}$ or $\lambda$-values depend explicitly on deformations and orientations of nuclei. Note that the only parameter of the model is the neck-length $\Delta R$, given in Table 1 , which decides the entry point of barrier penetration as well as the cluster's preformation.

Figure 3 shows the calculated (logarithms of) cluster decay half-lives, $\log _{10} T_{1 / 2}(s)$ for the most probable ${ }^{83} \mathrm{As}$ cluster emitted from various parent nuclei. The choice of cluster is based on the minima in the fragmentation potentials $V_{R}(\eta)$ [refer Eq. (5)] and hence for the cases of largest preformation factors $P_{0}$, illustrated as an example for parent nucleus ${ }^{289} 115$ in Figure 2. Calculations are made by using the PCM, taking the $Q$-value from Refs. $[14,15]$ for spherical and with $\beta_{2 i}$ alone having appropriate 'hot optimum' orientations. Also shown in Figure 3 are the results of another recent calculation by Poenaru et al. 
Table 1: The calculated preformation probability $P_{0}$, penetrability $P$, and assault frequency $v_{0}$ using PCM for ${ }^{83}$ As cluster emitted from various parents, for cases of (a) spherical and (b) $\beta_{2}$ alone deformed nuclei having "optimum" orientations.

\begin{tabular}{cccccccccc}
\hline \multirow{2}{*}{ Decay } & \multicolumn{4}{c}{ Spherical } & & \multicolumn{3}{c}{$\beta_{2}$ deformed } & Assault frequency \\
\cline { 2 - 3 } & $\Delta R$ & Preformation & Penetration & & $\Delta R$ & Preformation & Penetration & $v_{0}\left(s^{-1}\right)$ \\
& $(\mathrm{fm})$ & probability & probability & & $(\mathrm{fm})$ & probability & probability & $\left(S p h ., \beta_{2}\right)$ \\
\hline${ }^{287} 115 \rightarrow{ }^{83} \mathrm{As}+{ }^{204} \mathrm{~Pb}$ & 0.450 & $4.41 \times 10^{-24}$ & $2.64 \times 10^{-5}$ & & 0.960 & $2.60 \times 10^{-25}$ & $4.59 \times 10^{-4}$ & $1.79 \times 10^{21}$ \\
${ }^{288} 115 \rightarrow{ }^{83} \mathrm{As}+{ }^{205} \mathrm{~Pb}$ & 0.150 & $1.42 \times 10^{-23}$ & $2.58 \times 10^{-7}$ & & 0.950 & $1.15 \times 10^{-26}$ & $3.15 \times 10^{-4}$ & $1.77 \times 10^{21}$ \\
${ }^{289} 115 \rightarrow{ }^{83} \mathrm{As}+{ }^{206} \mathrm{~Pb}$ & 0.250 & $1.40 \times 10^{-21}$ & $1.50 \times 10^{-6}$ & & 0.980 & $4.09 \times 10^{-24}$ & $5.71 \times 10^{-4}$ & $1.76 \times 10^{21}$ \\
\hline
\end{tabular}

$[4,5]$ based on the Analytical Super Asymmetric Fission Model (ASAFM) with the binding energies for $Q$-values taken from the AME11 [18] experimental mass tables, as well as using the calculated LiMaZe01 [19] and KTUY05 [20] mass tables. We find that PCM calculated half lives for the ${ }^{83}$ As clusters in the ground state decay of ${ }^{289} 115$, ${ }^{288} 115$, and ${ }^{287} 115$ SHE elements (for both spherical and $\beta_{2 i}$ deformations) are in good agreement with the predictions of ASAFM except for the use of $Q$ value obtained by using LiMaZe 01 mass table. It is evident that $Q$ value of decay fragments play a crucial role to account for the clusterization process in superheavy region, which in turn seem to suggest that heavy particle radioactivity behaves similar to normal cluster emission process.

\section{Summary and Conclusions}

Summarizing, we have extended our study $[9,10]$ on cluster decays of heavy parent nuclei to analyze the role of deformations in the ground state clusterization of the isotopes of SHE $Z=115$, using the Preformed Cluster Model. Apart from deformations, the comparison of hot (compact) and cold (non-compact) orientations is also analyzed. The results of the present calculations, using Audi-Wapstra and Möller Nix binding energies, are in good agreement with other predicted decay half-life times of ${ }^{83}$ As cluster emitted from ${ }^{289} 115,{ }^{288} 115$, and ${ }^{287} 115$ parent nuclei and thus are expected to provide a useful guideline for future experiments. The role of higher order deformations up to hexadecapole could be of further interest in reference to the heavy-particle radioactivity in super heavy region.

\section{Acknowledgement}

Financial support of the University Grants Commission, under Dr. D. S. Kothari program is duly acknowledged.

\section{References}

[1] A. Săndulescu, D. N. Poenaru, and W. Greiner, Sov. J. Part. Nucl. 11, 528 (1980).
[2] H. J. Rose and G. A. Jones, Nature (London) 307, 245 (1984).

[3] R. Bonetti and A. Guglielmetti, Romanian Reports in Phys. 59, 301 (2007).

[4] D. N. Poenaru, R. A. Gherghescu and W. Greiner, Phys. Rev. Lett. 107, 062503 (2011).

[5] D. N. Poenaru, R. A. Gherghescu, and W. Greiner, Phys. Rev. C 85, 034615 (2012).

[6] S. S. Malik and R. K. Gupta, Phys. Rev. C 39, 1992 (1989).

[7] G. Sawhney, M. K. Sharma and R. K. Gupta, Phys. Rev. C 83, 064610 (2011).

[8] Yu. Ts. Oganessian, et al. Phys. Rev. C 87, 014302 (2013).

[9] S. K. Arun, R. K. Gupta, B. B. Singh, S. Kanwar, and M. K. Sharma, Phys. Rev. C 79, 064616 (2009).

[10] S. K. Arun, R. K. Gupta, S. Kanwar, B. B. Singh, and M. K. Sharma, Phys. Rev. C 80, 034317 (2009).

[11] R. K. Gupta and W. Greiner, Int. J. Mod. Phys. E 3, 335 (Suppl., 1994).

[12] R. Bonetti and A. Guglielmetti, in Heavy Elements and Related New Phenomena, edited by W. Greiner and R. K. Gupta (World Scientific, Singapore, 1999), Vol. II, p. 643.

[13] R. K. Gupta, et al. J. Phy. G: Nucl. Part. Phys. 31, 631 (2005).

[14] G. Audi, A. H. Wapstra, and C. Thibault, Nucl. Phys. A 729, 337 (2003).

[15] P. Möller, J. R. Nix, W. D. Myers, and W. J. Swiatecki, At. Data Nucl. Data Tables 59, 185 (1995).

[16] R. K. Gupta, N. Singh, and M. Manhas, Phys. Rev. C 70, 034608 (2004).

[17] W. D. Myers and W. J. Swiatecki, Phys. Rev. C 62, 044610 (2000).

[18] G. Audi and W. Meng, (un-published).

[19] S. Liran, A. Marinov, and N. Zeldes, Phys. Rev. C 62, 047301 (2000); Phys. Rev. C 66, 024303 (2002).

[20] H. Koura, T. Tachibana, M. Uno, and M. Yamada, Prog. Theor. Phys. 113, 305 (2005). 